Objectives: We aimed to create an AR application that has informative content designed to educate users on the topics of basic brain anatomy and function. Furthermore, we aimed to increase the users understanding of the complete impairment of fatigue by creating a short video that describes living with fatigue from the patient's perspective.

Methods: The application was created using medical scan dataset, a variety of 3D modelling software, and a game engine to create a functional and interactive augmented application. The short video regarding a patient's perspective on living with fatigue was developed in collaboration with the Glasgow Arthritis Involvement Network patient partners. In order to determine if the application met its primary objectives a pilot test was conducted on 14 participants. After consenting to taking part in the study, individuals were guided through a pre-application test, the use of the application itself and finally a post-application test.

Results: Initial results from the pilot test showed promise in the educational potential of the application. With regards to the questions pertaining to the brain anatomy, the percentage of questions answered correctly increased from 36\% in the pre-test to $60 \%$ in the post-test. Furthermore, after using the application the participants reported a significant increase in their confidence for their answers. An additional six questions ascertained a participants perceptions of fatigue. From these questions, the answer that was most significantly changed after use of the application, was in relation to the impact that fatigue has on a patient's quality of life (t-Test $p=0.02)$. After use of the application participants' opinions changed to reflect the fact that fatigue can completely impair a person's quality of life, showing an increase in their understanding of the debilitating nature of fatigue.

Conclusion: This research explored the development and effectiveness of an AR application that was centered around fatigue and basic neuroanatomy education within the general population. From the pilot test conducted

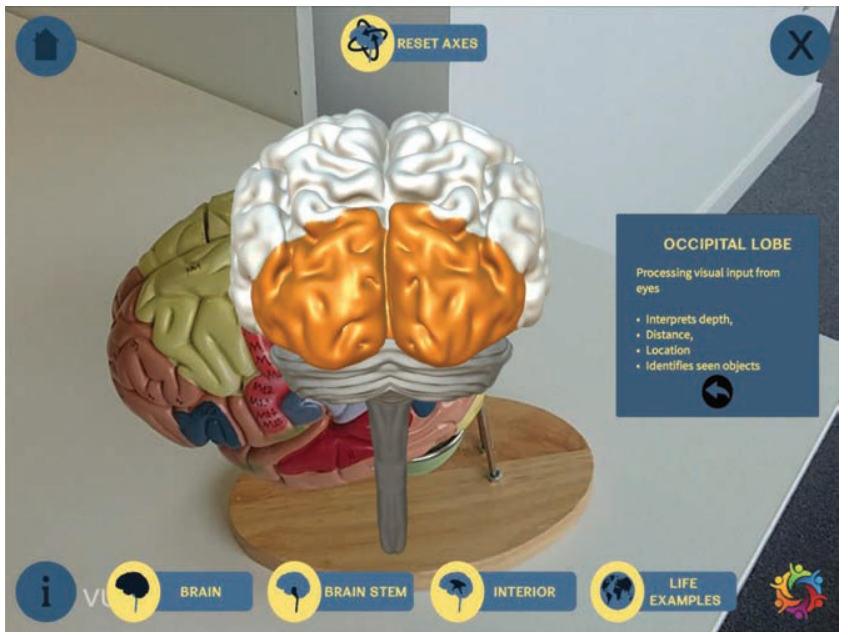

Figure 1. Augmented brain model scene using brain model as trigger

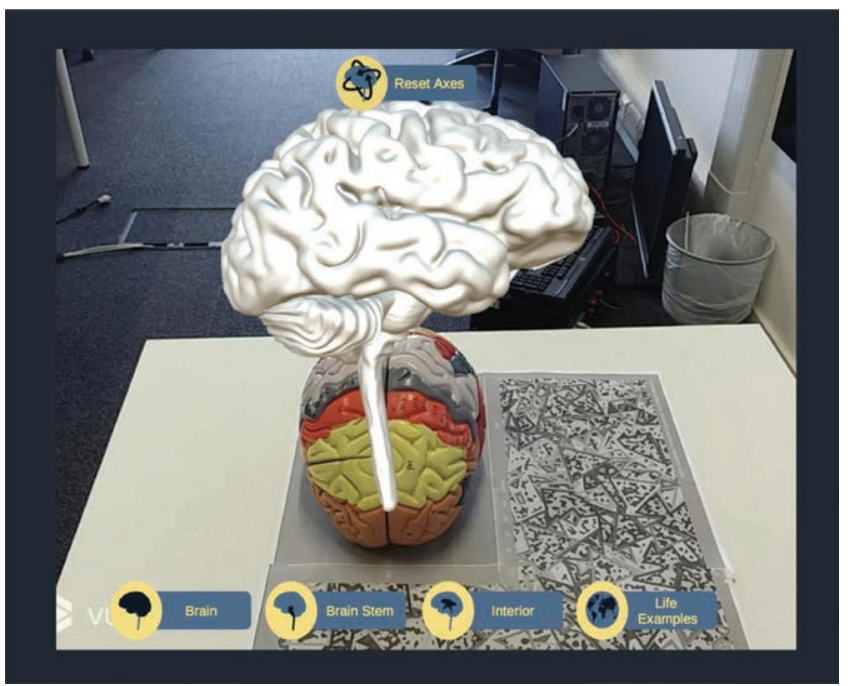

Figure 2. Example of material change upon selection of Occipital Lobe option we are able to report that the application was successful in delivering educational material about brain anatomy and was successful in increasing awareness about the impact that fatigue can have on an individual's quality of life.

Acknowledgments: The Glasgow Arthritis Involvement Network (GAIN)

Disclosure of Interests: None declared

DOI: 10.1136/annrheumdis-2020-eular.5342

\section{OP0256-PARE A PAW? YES, THANK YOU AN ANIMAL ASSISTED INTERVENTION (AAI) PILOT PROJECT FOR CHILDREN} WITH JUVENILE IDIOPATHIC ARTHRITIS (JIA)

U. Viora*1, P. Ponzio ${ }^{2}$, M. T. Mascarino ${ }^{3}$, B. Picco ${ }^{4}$, P. Guiso ${ }^{4}$, E. Battista ${ }^{4}$, S. Martino ${ }^{5}$, D. Montin ${ }^{6}$, M. Dellepiane ${ }^{6}$, G. Rosso $0^{6} .{ }^{1}$ AMaR Piemonte, Chieri, Italy; ${ }^{2}$ University of Turin, Veterinary Science, Grugliasco, Italy; ${ }^{3}$ AMaR Piemonte, Torino, Italy; ${ }^{4}$ Centro Studi Te.CA., Chieri, Italy; ${ }^{5}$ University of Torino, Torino, Italy; ${ }^{6}$ AO Città della Salute e della Scienza - OIRM Hospital, Torino, Italy

Background: Animal-Assisted Interventions (AAI) is the new way to indicate what was previously known as "Pet Therapy", as activities can be done either with the conventional "pets" (dogs, cats and rabbits) or with horses and donkeys. Children with JIA have several problems in terms of adherence both due to the atavistic fear of the needle and due to nausea and vomiting - the most important side effects of Methotrexate - often since the 2-3 days before the assumption to immediately after it.

Sure that animals can help children to forget this fear and to avoid the psycological conditions which enhance nausea, for the first time in Italy (and probably in Europe) it was designed a specific AAl program for these children.

Objectives: To promote a general state of psycho-physical well-being in children and families about:

manage of therapy; reduce discomfort and anxiety caused by entering hospital; improve self-esteem and the response to the stress generated by the execution of therapy and disease management; strengthen communication and socialization; stimulate the affective area through the activities of animal care.

Methods: Dogs and cats are part of the recreational activities once a week in an equipped area in the OIRM Hospital (no alternative gateway was needed). Paediatric Rheumatologists selected two different groups of children: the first one ( 5 children in the pilot study) every 15 days; the second one ( 5 children) every month; the selection was made looking at the therapeutic scheme.

Every session, one hour, has 3 clearly distinct stages:

Welcome and organization: children say hello to dogs and cats, open the toolkits specifically designed for the intervention, express their state of mind and are encouraged to tell their own stories.

Therapy: parents prepare and inject the drug to their children under medical or Health Professional control without discontinuation of the activities with animals. Play and socializing: children are involved in petting and other activities with animals; they are also involved in manipulative activities (design, puppets shows, modelling clay, animal care, ball retriving, etc). This step has the aim to relieve stress and discomfort due to medical procedures.

Visual Analogic Scales (VAS) were part of the toolkit, to let the researchers evaluate the effects of the activity directly from the children experience.

For the first time, we will control also the animal health status and wellness condition monitoring behavioural parameters and salivary cortisol level during each session.

Results: The pilot project started in October 2019 and nowaday we closed 12 meetings, 4 on October, 4 on November, 3 on December and 2 on January, with the participation of 2 dogs (Golden and Labrador Retriever) and 1 cat (Devon Rex) in each one.

All children love to play with animals, seek their closeness at the time of therapy and enjoy playing all together with the dogs; no one cry or refuse therapy and, since the third session, no one has nausea before, during or after the injection. Parents have reached a certain level of confidence: they stay quietly in the waiting room or go away to have a drink or to run an errand (it becomes a moment of relaxing for them too).

Animals remain in healthy and wellness conditions during the activity.

Conclusion: These preliminary data seem that AAl to be useful in helping patients in JIA to overcome some problems related to their pathology.

Disclosure of Interests: UGO VIORA: None declared, Patrizia Ponzio: None declared, Maria Teresa Mascarino: None declared, Barbara Picco: None declared, Paolo Guiso: None declared, Eleonora Battista: None declared Silvana Martino: None declared, Davide Montin Speakers bureau: Not relevant for the topic, Marta Dellepiane: None declared, Germana Rosso: None declared

DOI: 10.1136/annrheumdis-2020-eular.4899 